Research Article

\title{
Intelligent Vehicle Automatic Stop-and-Go Task Based on Humanized Learning Control Model
}

\author{
Tianjun Sun, ${ }^{1,2}$ Zhenhai Gao, ${ }^{1,2}$ Fei Gao, ${ }^{2}$ Tianyao Zhang, ${ }^{2}$ Di Ji, ${ }^{3}$ and Siyan Chen $\mathbb{D}^{1,2}$ \\ ${ }^{1}$ College of Automotive Engineering, Jilin University, Changchun 130012, China \\ ${ }^{2}$ State Key Laboratory of Automotive Simulation and Control, Jilin University, Changchun 130012, China \\ ${ }^{3}$ College of Communication Engineering, Jilin University, Changchun 130012, China \\ Correspondence should be addressed to Siyan Chen; chensiyan@jlu.edu.cn
}

Received 29 September 2020; Revised 12 November 2020; Accepted 6 January 2021; Published 18 January 2021

Academic Editor: Songtao Lv

Copyright ( $\odot 2021$ Tianjun Sun et al. This is an open access article distributed under the Creative Commons Attribution License, which permits unrestricted use, distribution, and reproduction in any medium, provided the original work is properly cited.

The automatic stop-and-go task of intelligent vehicles can make the adaptive cruise control system achieve a full-speed range. However, the conventional design methods mostly focus on functional safety, without considering drivers' behaviors, thereby leading to a poor driving experience. To improve the situation, a humanized learning control model is used instead of mechanical switching logic. Therefore, first, the common characteristics of human drivers with different driving styles are found by analyzing real drivers' experiments. Then, the vehicle automatic starting function is designed based on iterative learning control with the fast Fourier transform for acceleration fitting. Next, the vehicle automatic braking function is designed based on dynamic time to collision. Finally, the simulation of the stop-and-go scenario is shown in CARSIM, and the real vehicle test is performed under the urban overpass driving condition. Results show that the proposed model can improve the humanization in the vehicle stop-and-go task.

\section{Introduction}

With the high pace of modern life, the increasing number of citizens will cause traffic congestion. Although conventional adaptive cruise control (ACC) systems can provide partial drive assistance, such systems could still be improved. On the one hand, most ACC systems may not cover the fullspeed range [1]. On the other hand, the current automatic stop-and-go control methods of vehicles do not consider drivers' behavior [2].

Research on stop-and-go tasks is presently one of the most important topics in the field of ACC systems. One of the reasons is that the conventional ACC will frequently withdraw from the system when the velocity is less than $30 \mathrm{~km} / \mathrm{h}$. Moreover, the velocity is very low to enable cruise control (CC) function [3]. Furthermore, the current control methods mostly focus on functional safety. However, the poor driving experience will be increasingly evident when drivers' behaviors are not considered. For instance, some drivers used to have a violent start, such as a sense of push the back, but the control logic may limit the acceleration by considering safety and comfort. The other drivers used to make a double brake when a vehicle in front suddenly slows down, but the control logic may provide a great deceleration such that the host vehicle can stop by only braking once. Therefore, when the driver is aware of the relative motion of the vehicle in the field of view, the driver will make a series of habitual actions to catch up with the target vehicle and keep a safe distance. Thus, these actions reflect the real drivers' driving behaviors in car following, but the existing decision-making algorithms or control methods are compliant with the established rules.

The challenges of the research comprehensively regard drivers' behavior characteristics, vehicle's dynamic characteristics, safety, and humanization in vehicle automatic control. Moreover, in this study, an automatic stop-and-go control method is designed by analyzing the real drivers' starting and braking behaviors based on an intelligent vehicle platform, as shown in Figure 1.

According to previous studies, the conventional control methods for vehicle stop-and-go scenario can be 


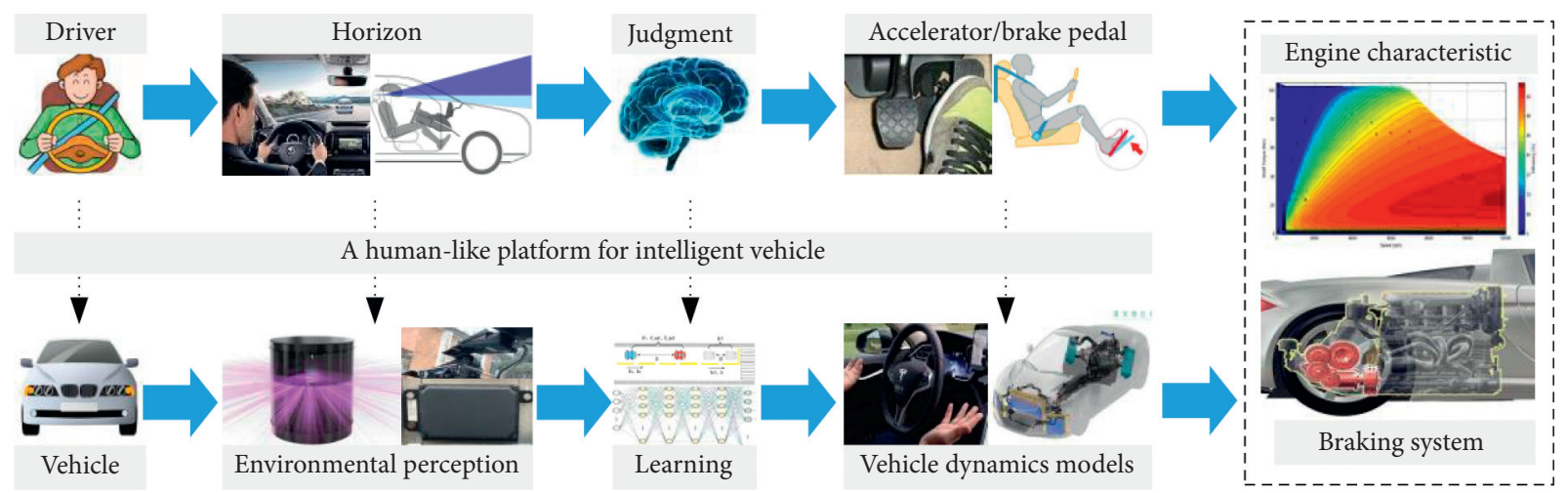

Figure 1: A human-like platform for intelligence vehicle.

summarized as model-based control. For example, Junchen [4] established a car-following model based on drivers' behavior and dynamic vehicle characteristics. However, model-based control methods are usually regarded as complicated and hard-to-debug systems. In the above methods, while also considering safety, the calculation of time to collision (TTC) plays an important role in the process of braking. As a quintessential example, Jahandideh et al. [5] found that pedestrians prefer to cross the road at the intersection point at the average TTC of approximately $6.2 \mathrm{~s}$, and $54 \%$ of men and $39 \%$ of women face TTC risk for less than $3 \mathrm{~s}$. Zhenhai and $\mathrm{Wu}[6,7]$ established models based on TTC for warning and braking time prediction on the basis of drivers' habitual braking and braking force characteristics. However, the conventional TTC algorithm cannot meet the change of acceleration. Compared with the conventional methods, some new trends toward learning control exist. For example, Lu et al. [8] designed a learning module based on neural reinforcement learning before the PID control. This model can learn from human drivers online based on the onboard sensing information and realize human-like longitudinal speed control by learning from the demonstration paradigm. Xing et al. [9] provided a personalized leading vehicle trajectory prediction method based on joint time series modeling. This method can generate three different driving styles for the leading vehicle through the JTSM algorithm and achieve better results. Lv et al. $[10,11]$ proposed a CPS-based framework for codesign optimization of an automated electric vehicle with different driving styles, which used unsupervised machine learning to develop the driving style recognition algorithm.

By analyzing the above results, this study finds that conventional methods are applicable, but humanization is not considered due to its inherent logic strategies. Therefore, based on previous studies, we propose a humanized vehicle automatic stop-and-go control method based on the iterative learning (IL) algorithm and the dynamic time to collision (DTTC) model. This study aims to develop a humanized learning control model based on IL and DTTC while focusing on the stop-and-go task for autonomous driving. The main contributions of this study are as follows: (1) the drivers' control behavior characteristics are extracted through the real vehicle tests with different types of drivers. (2) An automatic drive control method based on the IL algorithm is proposed to learn the driver behavior and realize the human-like control. Based on the IL algorithm, complex dynamic calculations are not required, and the control method can be learned directly from the acceleration curve equation. (3) An automatic brake control method based on the DTTC model is proposed in this study to learn the driver behavior and realize the human-like control. Based on the DTTC model, on the one hand, the safety of the braking process is improved by the consideration of dynamic deceleration; on the other hand, different types of braking can be learned directly from the real vehicle tests. (4) Automatic stop-andgo control is implemented by combining the automatic drive control and automatic brake control methods. The validity and feasibility of the proposed method are verified through simulation and real tests.

The remainder of this paper is organized as follows: the second part is "extraction of drivers' control behavior characteristics." The third part is the "learning model for vehicle automatic starting control." The fourth part is the "learning model for vehicle automatic braking control." The fifth part is "simulation and experimental tests." The sixth part is "results and comparative analysis." The final part is "conclusions and future work."

\section{Extraction of Drivers' Control Behavior Characteristics}

The three types of typical drivers are examined based on the previous classification to obtain the common characteristics of different drivers with evident driving styles [12-14]. In this study, we choose three types, namely, aggressive driver, normal driver, and steady driver. Many factors causing different characteristics should be neglected because this part aims to find the humanized common characteristics of human drivers compared with the conventional modelbased design. Therefore, we assume that the target vehicle stops at the stop line $200 \mathrm{~m}$ away from the host vehicle. Then, the host vehicle starts to catch up with the target vehicle through acceleration or deceleration control over a period of 
time. Finally, the host vehicle will stop behind the target vehicle. Figure 2 shows the experimental scenario.

As shown in Figure 3, we conduct further analysis of vehicle velocity and acceleration in the stop-and-go scenario. In the case of low speed, the vehicle stop-and-go characteristics controlled by different drivers have some similarities. One similarity is that the trend of the acceleration curve will increase along with the velocity and then gradually reduce to half of the peak value. From a practical point of view, the drivers in the experiment with different start characteristics usually choose to step down the accelerator pedal. Then, they slowly release the accelerator pedal in the process of starting. The other similarity is the time of braking. Although different drivers have varying braking decelerations, a humanized braking method is reflected in a reasonable double brake. Moreover, the trend of acceleration change is highly similar to the same velocity adjustment, and the trend of deceleration with a large increase or decrease can be classified as a double brake.

\section{Learning Model for Vehicle Automatic Starting Control}

When the vehicle deals with repetitive tasks in an actual environment, people expect to use a certain amount of human manipulation samples to match the automatic control system with the behavior of the drivers. To imitate human learning abilities and their self-regulation function, the IL algorithm can improve the control target through iterative correction, which is suitable for controlled objects with repetitive motion properties. The IL algorithm can realize the control of strong coupled nonlinear dynamic systems with high uncertainty in a limited time. Moreover, this algorithm does not depend on precise mathematical models $[15,16]$. Therefore, IL has been widely used in automation controls, such as industrial robots, digital control machines, and machine manufacturing.

However, in the practical environment, the driving styles of drivers are better described through behaviors but hard to describe through theoretical analysis. Therefore, in humanized features' extraction, we will transform the practical problem into a function approximation problem based on experimental data. Thus, the first step is to design a function to make it as close as possible to the trend of the acceleration curve.

According to previous studies, two methods are usually used for solving function approximation problems, namely, interpolation and fitting. If the experimental data are discrete, then the interpolation is suitable. When considering the continuity of the acceleration curve, the fitting is suitable instead. The three main methods of fitting that are used widely are algebraic polynomials, rational fractional functions, and triangular polynomials. Typical samples for the fitting methods above are polynomial fitting, Gaussian fitting, and fast Fourier transform (FFT). Moreover, we construct a function similar to the trend of the acceleration curve in the experiment, which will increase along with the velocity and then gradually reduce to half of the peak value. If we choose the polynomial power function for fitting, then estimating the error is difficult although easy to operate, and the amount of calculation is small. When the sampling points are rare, the fitting effect is poor, as shown in Figure 4(a). Otherwise, if we choose the Gaussian function for fitting, then the positioning accuracy for edge detection of data will be low, and the fitting will be sensitive to noise although calculating the integral is convenient, as shown in Figure 4(b).

Figure 4 shows that underfitting occurs in polynomial fitting, and overfitting occurs in Gaussian fitting. From the results above, these two methods for acceleration curve fitting can meet the approximation standard, but each has shortcomings. Therefore, FFT fitting is made by analyzing polynomial and Gaussian fitting methods to better reflect the humanization of acceleration in the vehicle stop-and-go scenario. FFT is not a change of discrete Fourier transform (DFT) but is a fast algorithm which can reduce the number of DFT operations. Among them, any periodic function can be converted into the sum of several trigonometric functions by dividing the periodic function to be converted $f(t)$ into a trigonometric function of innumerable sin and cos, as shown in the following equation:

$$
\begin{aligned}
f(t)= & \frac{1}{T} \int_{t_{0}}^{t_{0}+T} f(t) d t+\left[\frac{2}{T} \int_{t_{0}}^{t_{0}+T} f(t) \sin (\omega t) d t \sin (\omega t)+\frac{2}{T} \int_{t_{0}}^{t_{0}+T} f(t) \cos (\omega t) d t \cos (\omega t)\right]+, \ldots \\
& +\left[\frac{2}{T} \int_{t_{0}}^{t_{0}+T} f(t) \sin (n \omega t) d t \sin (n \omega t)+\frac{2}{T} \int_{t_{0}}^{t_{0}+T} f(t) \cos (n \omega t) d t \cos (n \omega t)\right]+, \ldots
\end{aligned}
$$

Furthermore, we use the toolbox of MATLAB to conduct the acceleration fitting based on FFT to the experimental data for vehicle starting, and Figure 5 shows the result.

The FFT fitting curve on level 4 can effectively express the fluctuation trend of the experimental data through comparative analysis. If we regard the FFT fitting curve on level 4 as the target acceleration that the system needs to follow, then the second step would be the vehicle automatic starting based on the IL algorithm.
Owing to the influence of several factors, such as external air resistance, road friction resistance, and internal transmission, the longitudinal acceleration of the vehicle and the torque of the engine have a nonlinear relationship. Furthermore, the control principle of the automatic vehicle start control algorithm is based on the engine torque control to track the target acceleration. Hence, to express the nonlinear relationship, this study uses the PD-type close-loop IL 


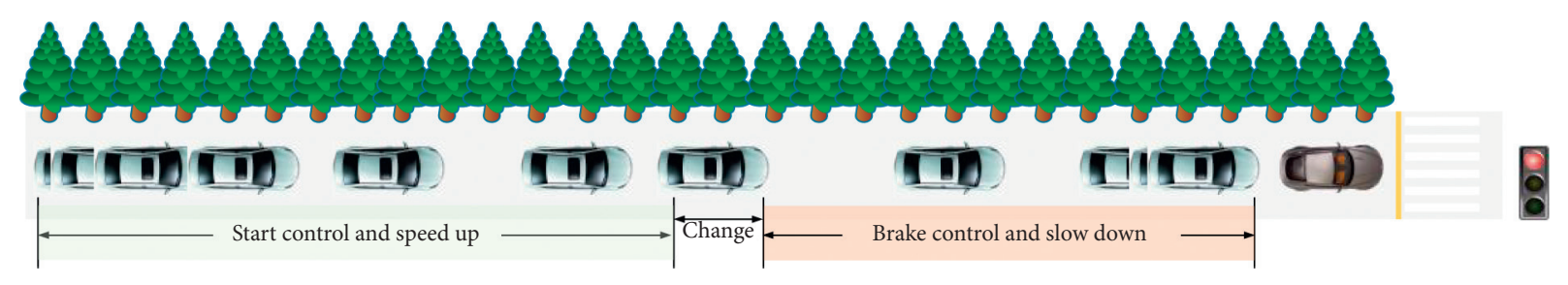

Figure 2: The experimental scenario.

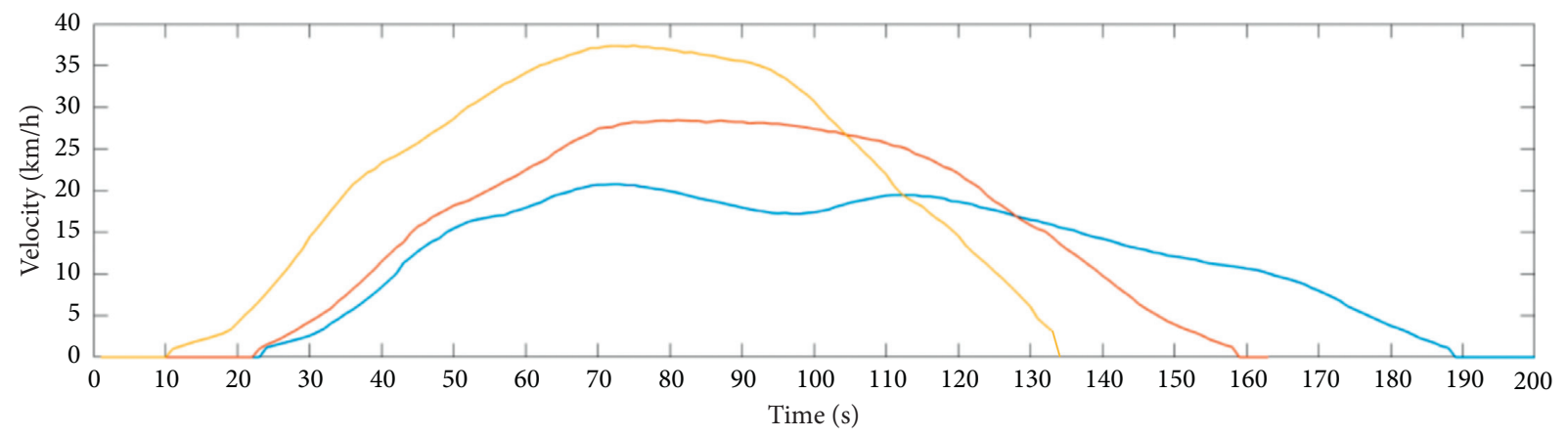

- An aggressive driver

- A normal driver

— A steady driver

(a)

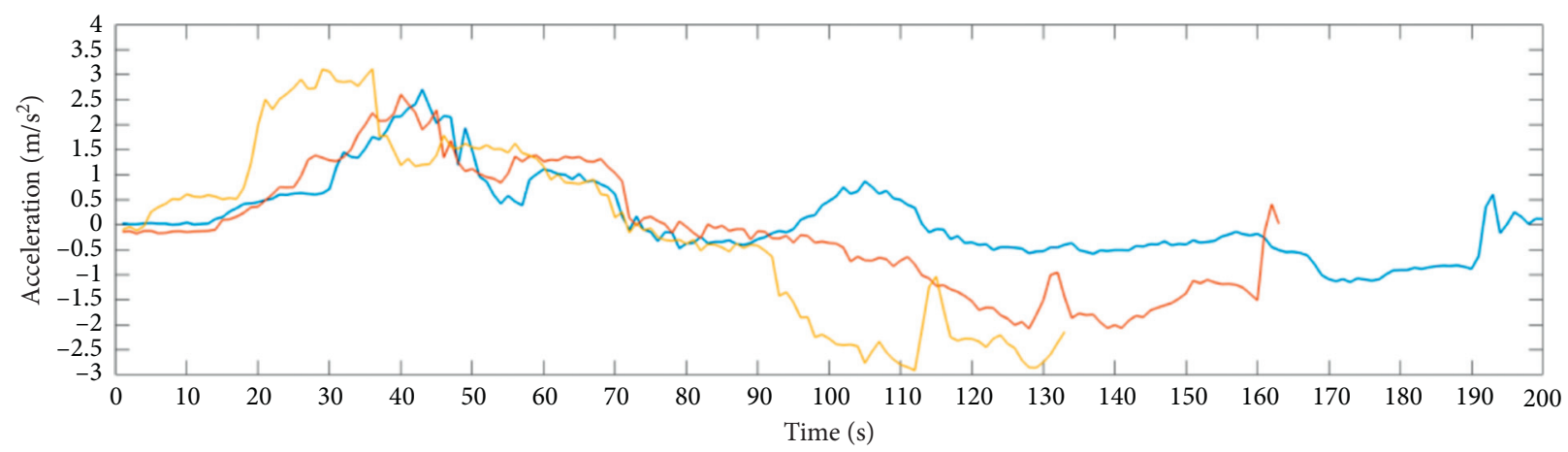

- An aggressive driver

- A normal driver

— A steady driver

(b)

FIgURE 3: The experimental results for three drivers in the vehicle stop-and-go scenario.

control method to convert the engine torque control problem into an engine throttle opening problem. The IL algorithm can be described as shown in the following equation:

$$
\left\{\begin{array}{l}
p_{k}(t)=p_{k-1}(t)+L_{p} e_{k}(t)+L_{d} e_{k}(t) \\
e_{k}(t)=a_{\text {expected }}(t)-a_{\text {real }}(t)
\end{array}\right.
$$

where $p_{k}(t)$ represents the throttle percentage, $a_{\text {expecred }}(t)$ represents the expected acceleration, $a_{\text {real }}(t)$ represents the real acceleration, $L_{p}$ and $L_{d}$ represent the learning gain factors, and $e_{k}(t)$ represents the error.

As mentioned before, in this study, a typical fitting function is extracted from the experimental test data to be used as the target acceleration for tracking the target of IL control, as shown in Figures 6 and 7.

In Figure 6, the error gradually decreases with the increase of the iteration number, and when the iteration reaches the eighth time, the error is close to 0 . Therefore, in Figure 7 , the acceleration following the curve from the first iteration to the eighth iteration and the throttle change curve is provided. Figure 7 (a) shows that with the increase of iteration time, the actual acceleration of the vehicle draws closer to the target acceleration and converges to the expected trajectory. In addition, Figure 7(b) shows that with the increase of the number of iterations, the control quantity gradually converges to the expected control quantity and converges to the expected trajectory. 


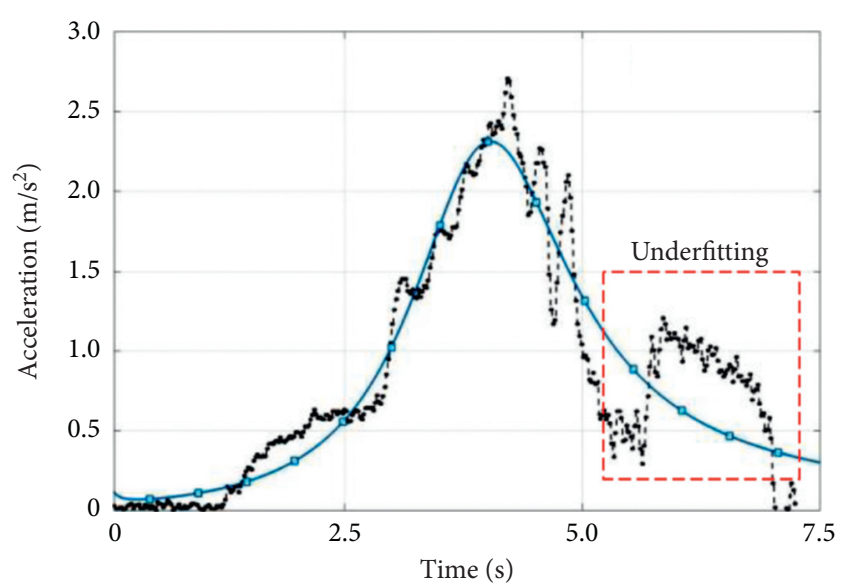

-.... Experimental data curve

_ Polynomial fitting curve

(a)

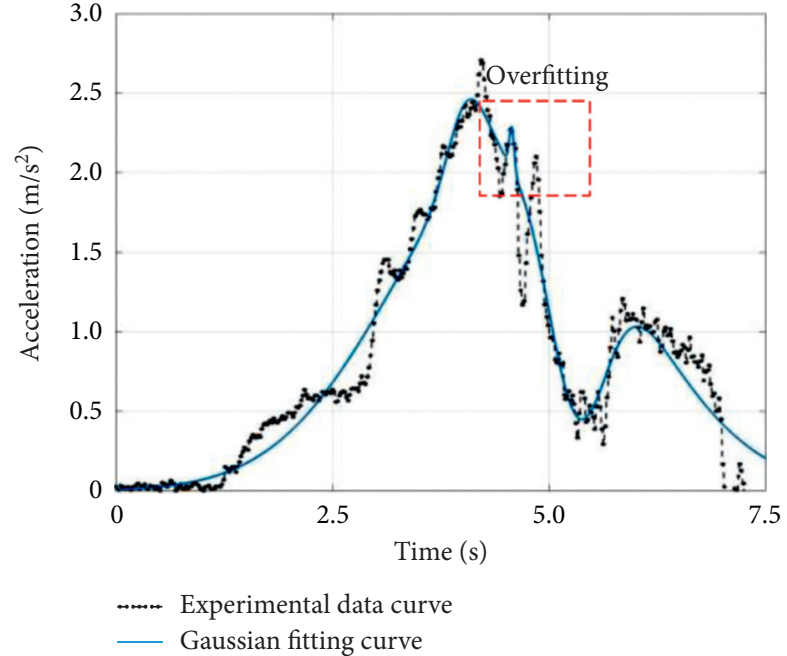

(b)

Figure 4: Acceleration fitting based on different fitting methods. (a) Polynomial fitting. (b) Gaussian fitting.

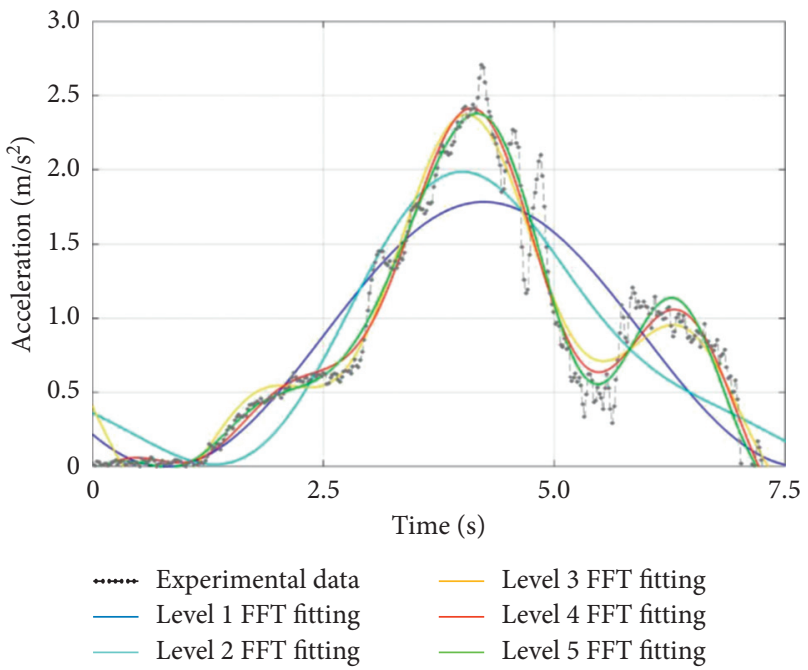

Figure 5: Acceleration fitting based on multiple FFT method.

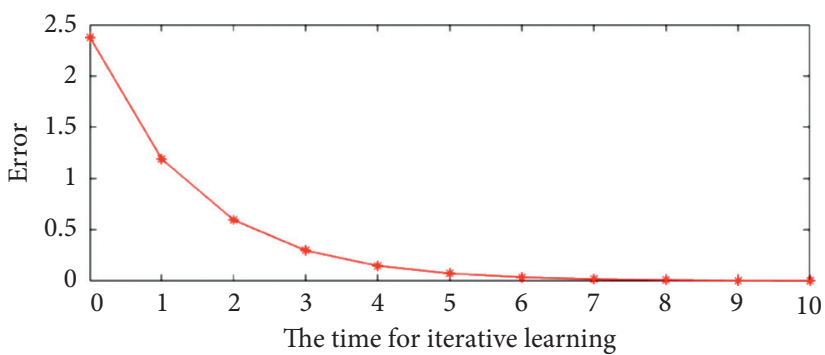

FIgURE 6: The error varies with the time for iteration learning. 


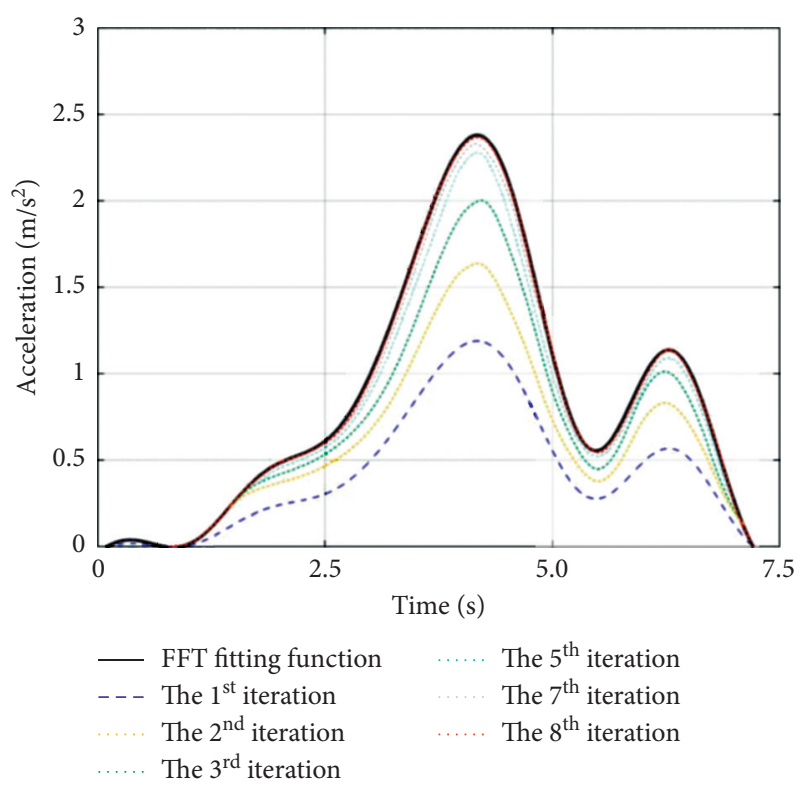

(a)
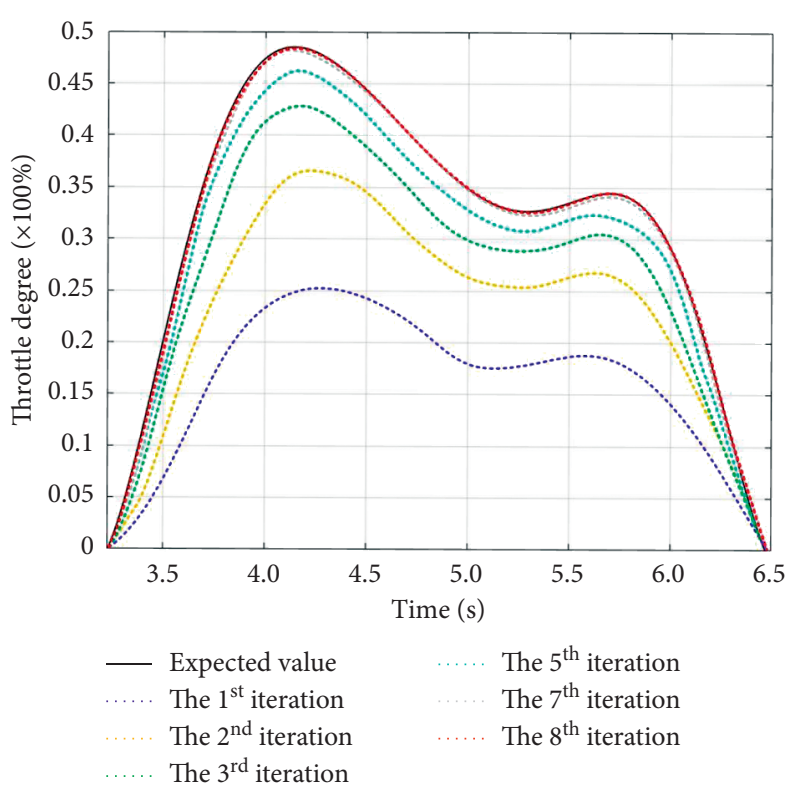

(b)

FIGURE 7: The results of the vehicle automatically start based on the IL control method. (a) Acceleration following with multiple iterations. (b) Throttle change with acceleration following.

\section{Learning Model for Vehicle Automatic Braking Control}

The vehicle automatic braking control is an active method to apply braking force through the electronic stability program before the collision happens. Safety should be prioritized to ensure that the host vehicle can slow down when the target vehicle decelerates or suddenly brakes when car following, which is different from the starting control. According to previous studies [17-19], the cause of the collision is related to different drivers' braking time, braking behavior, and braking force. The design of the conventional vehicle automatic braking control method only considers the rigid body kinematics characteristics and collision theory. Therefore, the driver and the passenger will feel an evident sense of frustration and tension due to the lack of understanding of the driver's braking characteristics.

At present, classification judgment is a popular method widely used in the vehicle longitudinal automatic braking control. This method is based on the comprehensive information of vehicle and traffic conditions. As the danger comes, the vehicle will slow down through active intervention. However, in this study, we aim to consider the uncertainty of the target vehicle and propose an anthropomorphic automatic braking control method based on the DTTC model. Thus, through the analysis of Figure 3, the humanized design problem is transformed into the interpretation of brake commonality.

As previously mentioned, the basic requirement of the vehicle automatic braking control is to achieve driving safety. The conventional calculation method for TTC is shown in the following equation:

$$
\mathrm{TTC}=\frac{D_{\text {rel }}}{V_{\text {rel }}}
$$

where $D_{\text {rel }}$ and $V_{\text {rel }}$ represent the relative distance and velocity between the vehicles, respectively. However, this definition of TTC does not consider the speed change of the ego vehicle and target vehicle during acceleration and deceleration. Therefore, as shown in Figure 8, DTTC is a definition of a collision which considers the host vehicle's velocity $v_{\text {ego }}$ and deceleration $a_{\text {ego }}$ and the target vehicle's velocity $v_{\mathrm{tar}}$ and deceleration $a_{\mathrm{tar}}$.

Based on the kinematic relation of the vehicle, the value of DTTC can be obtained as shown in the following equation:

$$
\begin{aligned}
& D_{\text {ego }}=V_{\text {ego }} t+\frac{1}{2} a_{\text {ego }} t^{2}, \\
& D_{\text {tar }}=V_{\mathrm{tar}} t+\frac{1}{2} a_{\mathrm{tar}} t^{2}, \\
& D_{\text {ego }}=D_{\text {rel }}+D_{\text {tar }}, \\
& V_{\text {rel }} t+\frac{1}{2} a_{\mathrm{tar}} t^{2}+D_{\text {rel }}=0 .
\end{aligned}
$$

Moreover, through the further discussion on $v_{\text {rel }}$ and $a_{\text {rel }}$, the DTTC can be described as shown in the following equation: 


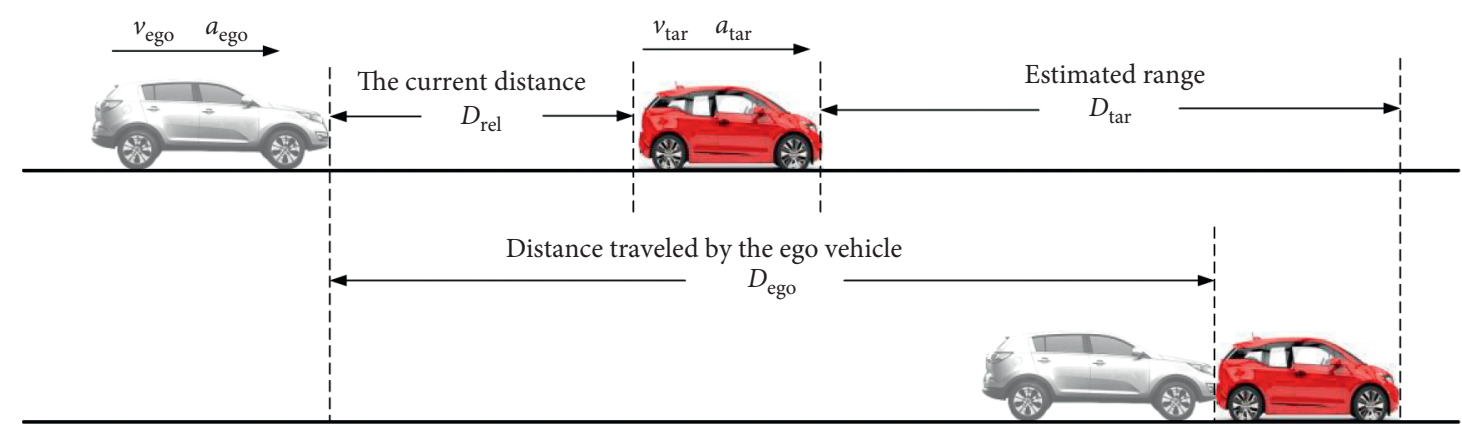

FIgURE 8: The condition of the DTTC process.

DTTC $=\left\{\begin{array}{cl}\infty, & V_{\text {rel }}<0 \text { and } a_{\text {rel }}<0, \\ \frac{\left(-V_{\text {rel }}\right)-\sqrt{V_{\text {rel }}^{2}-\left(2 a_{\text {rel }} D_{\text {rel }}\right)}}{a_{\text {rel }}}, & V_{\text {rel }}<0 \text { or } a_{\text {rel }}<0 .\end{array}\right.$

An ideal braking model based on the minimum safe distance should be considered to make the deceleration process of vehicle automatic braking control reflect the characteristics of the driver's behavior. In Figure 8, on the one hand, the ideal braking model provides an expected deceleration in the process of slowing down, which can be defined as $a_{\text {req }}$ represents the deceleration avoiding a collision. On the other hand, the host vehicle should maintain a minimum safe distance from the target vehicle after braking, which can be defined as $D^{*}$, as shown in Figure 9. Furthermore, the following equation provides the calculation of $a_{\text {req }}$ :where $D_{\text {rel }}$ represents the current distance, $D_{\text {tar }}$ represents the estimated distance, and $D_{\text {ego }}$ represents the distance the host vehicle traveled.

$$
\begin{aligned}
D_{\text {ego }} & =V_{\text {ego }} t+\frac{1}{2} a_{\text {req }} t^{2}, \\
D_{\text {ego }}+D^{*} & =D_{\text {rel }}+D_{\text {tar }}, \\
a_{\text {req }} & =\left\{\begin{array}{cc}
0, & V_{\text {rel }}>0 \text { and } a_{\mathrm{tar}}>0, \\
\frac{V_{\text {ego }}^{2}}{\left(V_{\mathrm{tar}}^{2} / a_{\mathrm{tar}}\right)-2^{*}\left(D_{\mathrm{rel}}-D^{*}\right)}, & V_{\mathrm{rel}}>0 \text { and } a_{\mathrm{tar}}<0, V_{\mathrm{rel}}<0 \text { and } a_{\mathrm{tar}}<0 \text { and } t_{\mathrm{ego}}>t_{\mathrm{tar}}, \\
a_{\mathrm{tar}}-\frac{V_{\mathrm{rel}}^{2}}{2^{*}\left(D_{\mathrm{rel}}-D^{*}\right)}, & V_{\mathrm{rel}}<0 \text { and } a_{\mathrm{tar}}>0, V_{\mathrm{rel}}<0 \text { and } a_{\mathrm{tar}}<0 \text { and } t_{\mathrm{ego}}<t_{\mathrm{tar}},
\end{array}\right.
\end{aligned}
$$

\section{Simulation and Experimental Tests}

A simulation test based on CARSIM and a real vehicle test under the urban overpass driving condition are provided to verify the validity and feasibility of the proposed method. In this part, we first establish a vehicle longitudinal automatic control model in MATLAB/SIMULINK. This model contains car, sensor, decision-making, and execution modules, as shown in Figure 10.

The car module is connected to CARSIM, which provides some vehicle, environmental, and road parameters. The sensor module is connected between CARSIM and SIMULINK, which provides information for the host and target vehicles. The decision-making module is designed on MATLAB/SIMULINK, which provides the key strategies and important logics for the vehicle autonomous stop-and-go task. The execution module is a computational relationship between vehicle dynamics and the braking system. Then, we conducted a simulation test for the vehicle autonomous stop-and-go scenario through CARSIM/SIMULINK, as shown in Figures 11 and 12 .

To further verify the proposed method, a real vehicle test is conducted. The experimental vehicle is HAVAL H7 equipped with a millimeter wave radar and dSPACE AutoBox. The former is used to obtain the information of the target vehicle, whereas the latter is used to obtain the information of the host vehicle. Moreover, we download the control algorithm to dSPACE AutoBox instead of the original controller of the vehicle. Therefore, a real vehicle test is performed under the urban overpass driving condition, as shown in Figures 13 and 14. 


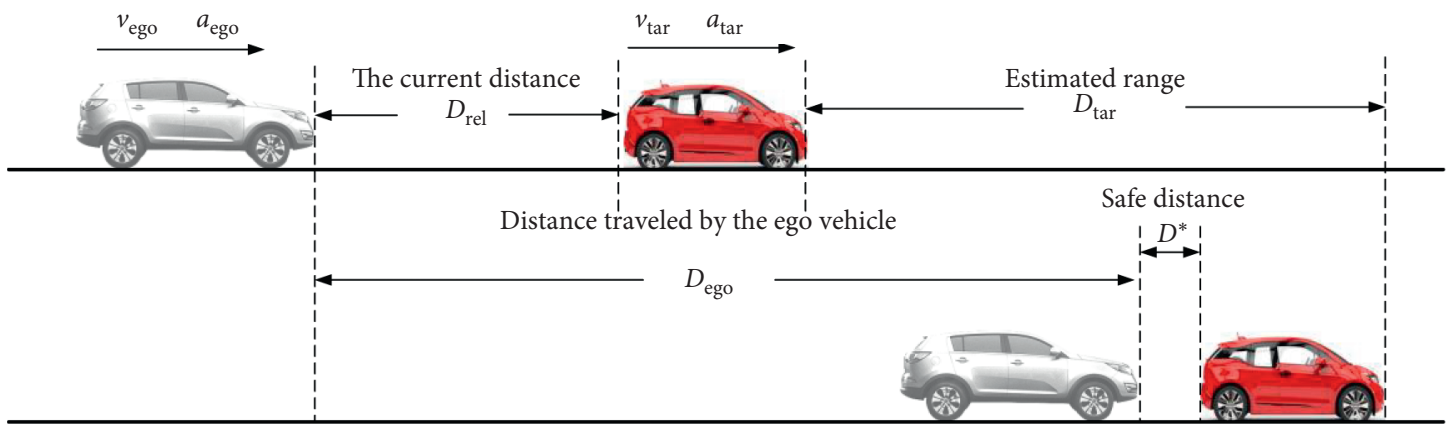

FIGURE 9: The ideal braking model based on DTTC.

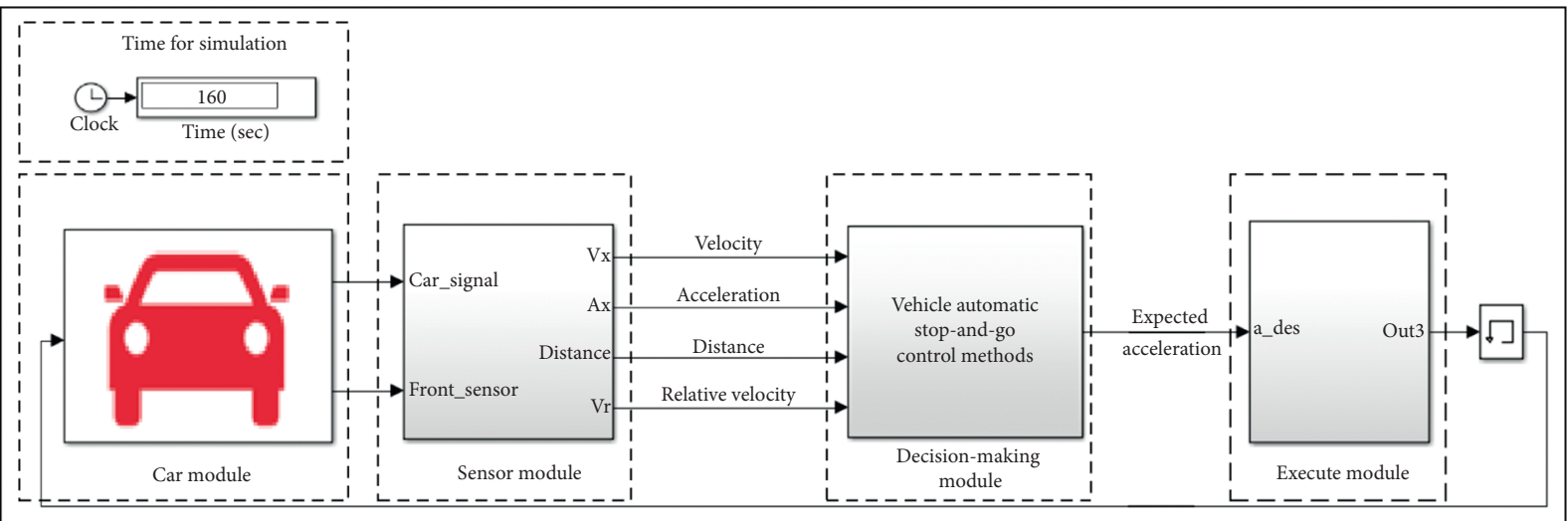

FIGURE 10: The vehicle longitudinal automatic control model based on MATLAB/SIMULINK.

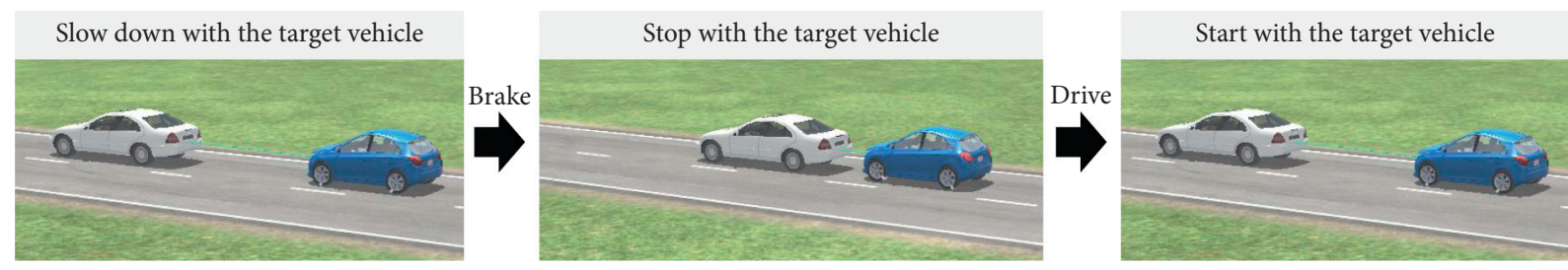

FIGURE 11: The vehicle autonomous stop-and-go task in CARSIM.

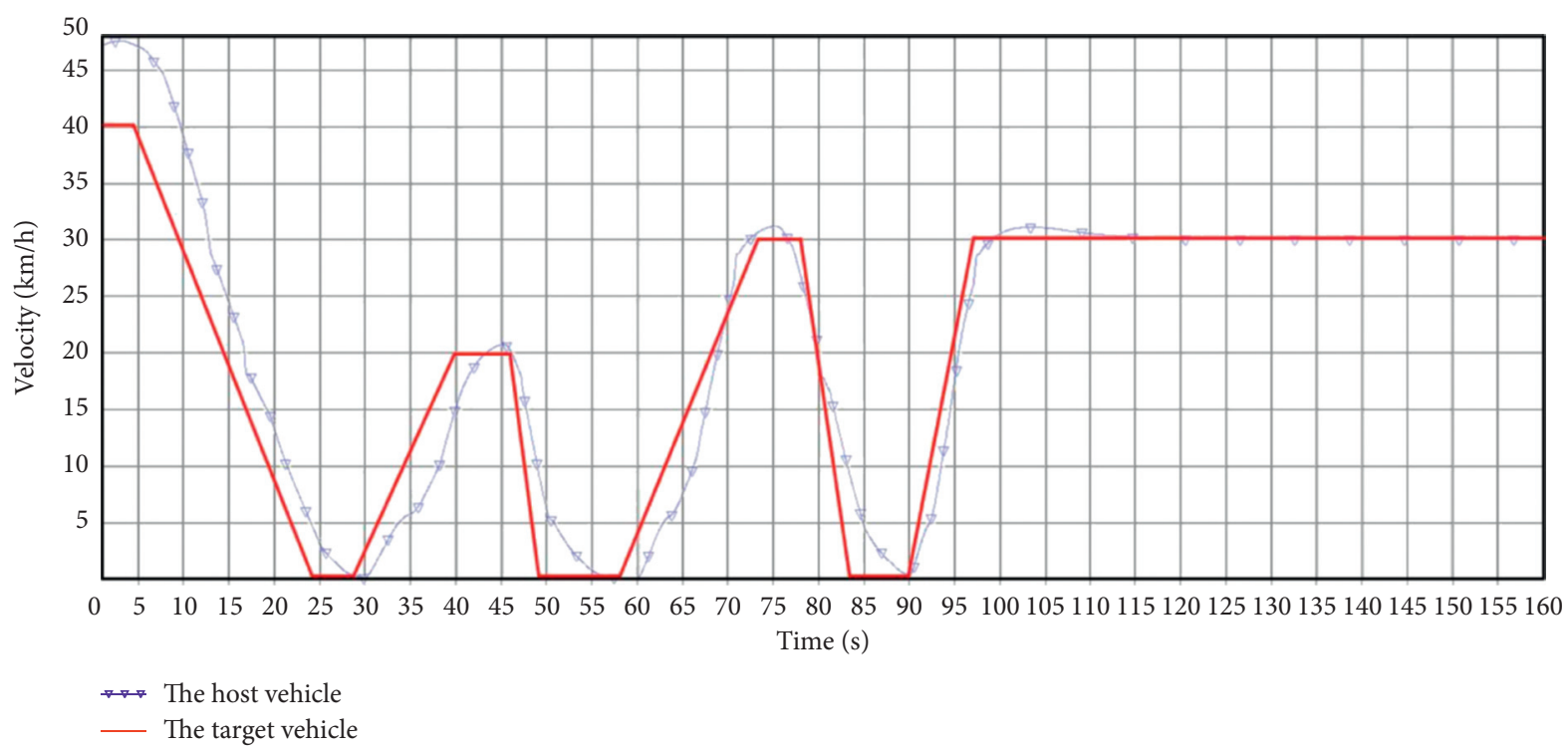

(a)

Figure 12: Continued. 


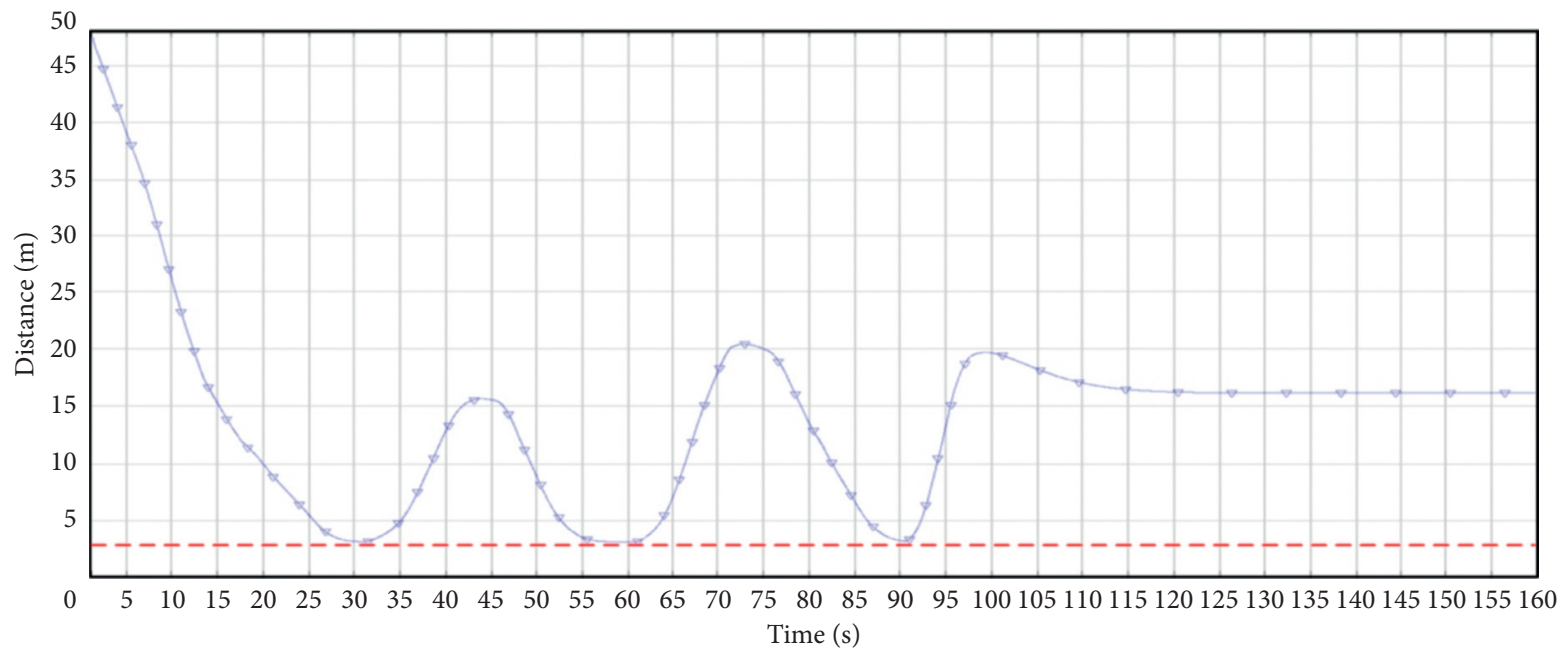

$\rightarrow$ Distance between two vehicles

-- An ideal safe distance $\left(\mathrm{D}^{*}=3.5 \mathrm{~m}\right)$

(b)

FIgURE 12: The simulation results for vehicle automatic stop-and-go control. (a) The simulation results for velocity in vehicle stop-and-go scenario. (b) The simulation results for distance in vehicle stop-and-go scenario.
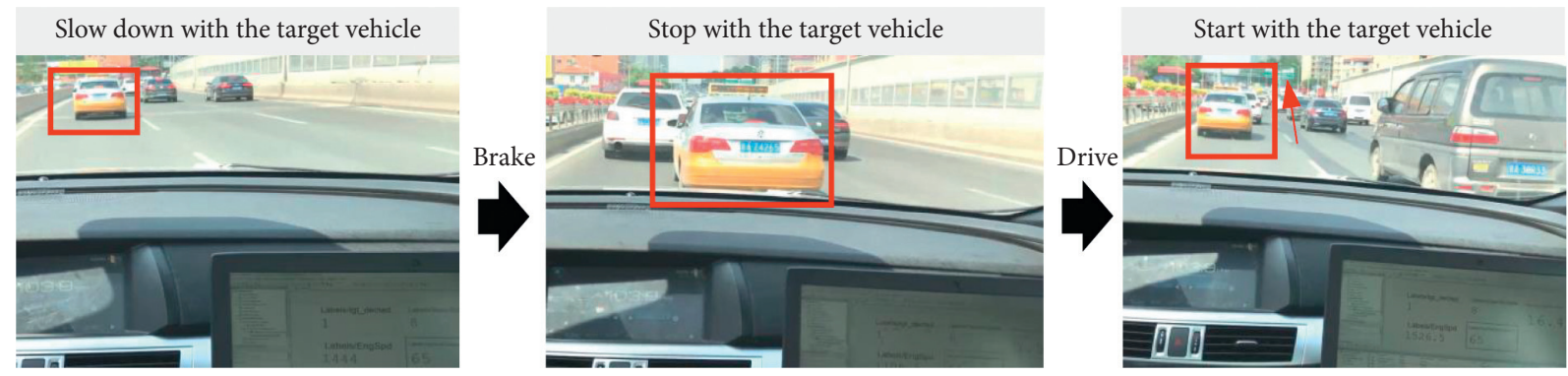

FIGURE 13: The real vehicle automatic stop-and-go task under the urban overpass driving condition.

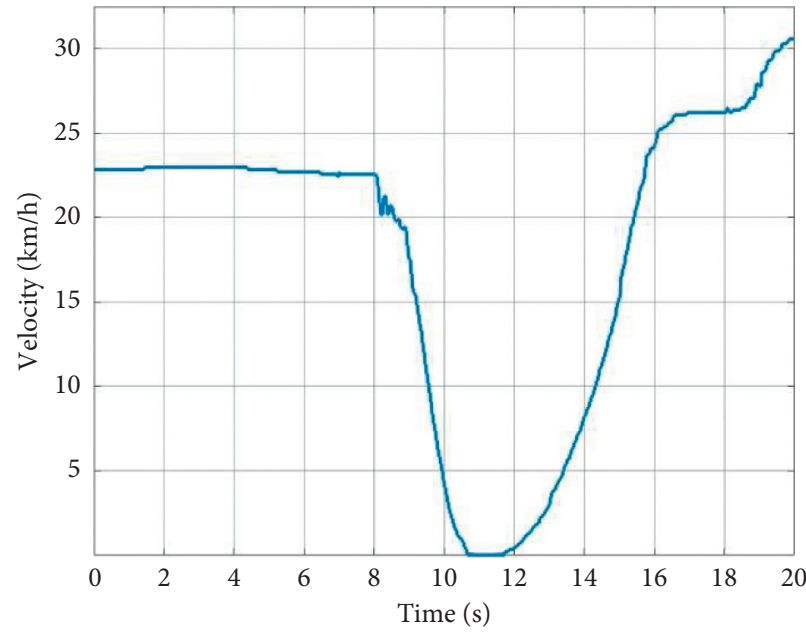

(a)

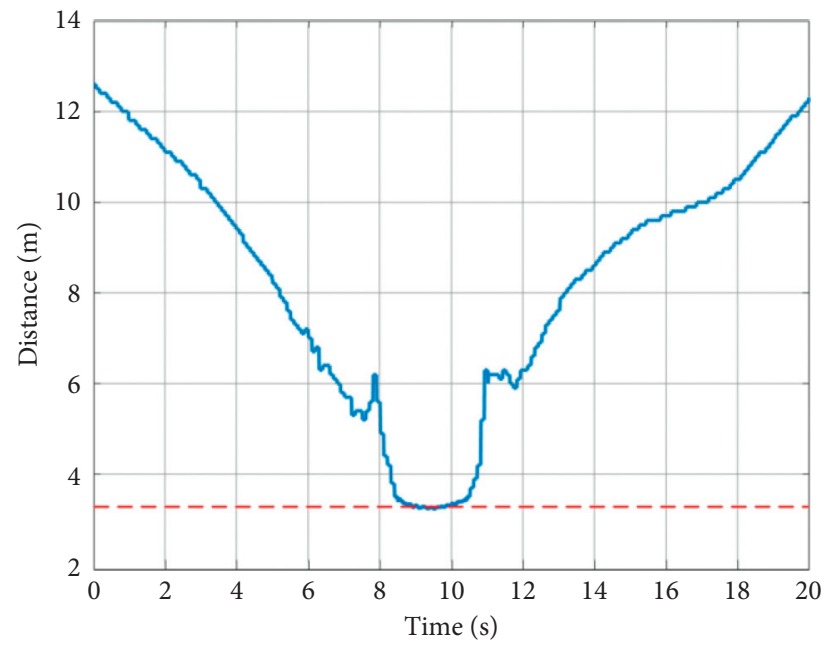

- Distance between two vehicles

-- An ideal safe distance $\left(\mathrm{D}^{*}=3.5 \mathrm{~m}\right)$

(b)

Figure 14: The real vehicle automatic stop-and-go task under the urban overpass driving condition. (a) The host vehicle's velocity. (b) The distance between two vehicles. 


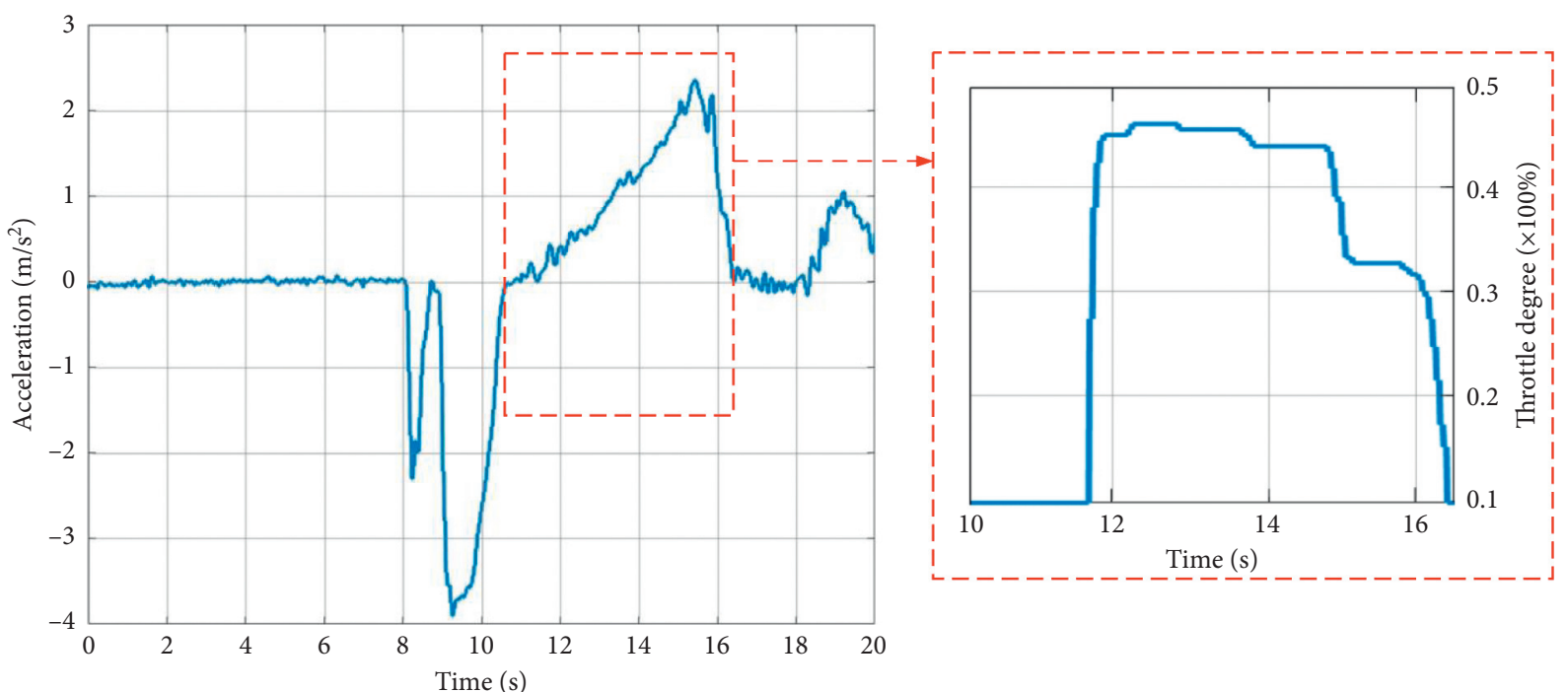

FIgURE 15: The acceleration and throttle degree for the real vehicle experimental test.

\section{Results and Comparative Analysis}

During the simulation, as shown in Figure 12, the driving condition is set to realize the vehicle's frequent stop-and-go task. The results show that the host vehicle can catch up with the target vehicle and keep an ideal distance when car following. Moreover, as shown in Figure 14, the experimental condition is random under urban overpass. The results show that the host vehicle can achieve an autonomous stop-and-go control based on the proposed method when the target vehicle is accelerating or decelerating. However, to further illustrate humanization, a comparative analysis is conducted, as shown in Figure 15.

The trend of acceleration is similar to the trend in Figure 7(a). Moreover, the trend of throttle degree is also similar to the trend in Figure 7(b). Therefore, on the one hand, the sense of starting in the real vehicle's experimental test will be similar to the real drivers. On the other hand, with the evident change in double deceleration, the behavior of braking will also be similar to the real drivers. Furthermore, the ideal distance in the simulation is similar to the test, and, then, the effectiveness of the proposed method is verified on the premise of ensuring safety.

\section{Conclusions and Future Work}

In this article, we propose an automatic stop-and-go control method based on a learning model for vehicles. First, the real drivers' starting and braking behaviors are obtained through the real vehicle's experimental test. The results show that the common characteristics of human drivers with different driving styles are their humanized acceleration and deceleration. Then, according to the variation trend of acceleration, the vehicle automatic starting control strategy is designed based on FFT and the IL algorithm. Next, based on the DTTC model, the vehicle automatic braking control strategy is designed by further analyzing the common humanized characteristics. Finally, the validity and feasibility are proved through the simulation and real vehicle tests. Furthermore, compared with the initial experiment, the method proposed can provide automatic stop-and-go control in car-following and improve the sense of humanity in the vehicle stop-and-go task.

However, the humanized learning control method has still some limitations: (1) considering the limited sample size, the humanization of the algorithm is slightly inadequate. (2) A switch logic should be designed between drive and brake controls. (3) The proposed method does not consider some extreme conditions, such as emergency braking behavior.

Future work can increase the sample size of the learning model. In addition, the switch strategy can be improved to solve the fluctuation problem, and some extreme conditions may be tested in the simulation.

\section{Data Availability}

The data used in the paper are obtained through actual experiments, rather than using the established experimental data. Among them, some data or curves were derived from previous research results, which have been presented at a conference in 2019 Chinese Automation Congress (CAC); https://ieeexplore.ieee.org/document/8996633).

\section{Disclosure}

This study is a continuation of the previous work, which has been presented at a conference in 2019 Chinese Automation Congress (CAC; https://ieeexplore.ieee.org/document/ 8996633).

\section{Conflicts of Interest}

The authors declare no potential conflicts of interest with respect to the research, authorship, and/or publication of this article. 


\section{Acknowledgments}

This work was supported by National Science Foundation of China (Grants 51775236, 51675224, and U1564214), National Key Research and Development Program (Grant 2017YFB0102600), and Science and Technology Development Project of Jilin province (20200501012GX). The authors gratefully acknowledge Zhenhai Gao, Fei Gao, Tianyao Zhang Ji Di, and Siyan Chen of Jilin University for their helpful discussion.

\section{References}

[1] Z. Li, Research on Full Speed Adaptive Cruise Control System of Pire Electric Vehicle, Harbin Institute of Technology, Harbin, China, 2017.

[2] L. Yishi, Z. Zhe, W. Liqiang, and H. Zongqi, "Design of vehicle full-speed adaptive cruise control with stop and go," $\mathrm{Ma}$ chinery Design and Manufacture, vol. 5, pp. 174-177, 2017.

[3] V. Paul, N. Karl, and A. Bartono, Stop and Go Cruise Control, FISITA World Automatic Congress, Seoul, Korea, 2000.

[4] J. Junchen, "Improved analysis of the car-following model based on ACC system," Science, Technology and Engineering, vol. 11, no. 26, pp. 6396-6400, 2011.

[5] Z. Jahandideh, B. Mirbaha, and A. A. Rassafi, "Modelling the risk intensity of crossing Pedestrians in intersections based on selected critical time to collision: a case study of Qazvin city," Transportation Research Board 96th Annual Meeting, vol. 1, 2017.

[6] G. Zhenhai, "Vehicle virtual following collision avoidance driver braking time model," Journal of Jilin University (Engineering Edition), vol. 44, no. 5, pp. 1233-1239, 2014.

[7] T. Wu, "A Study on Forward Collision Prevention System Considering the Characteristics of the Driver's Collision Avoidance Behavior, Jilin University, Changchun, China, 2014.

[8] C. Lu, J. Gong, C. Lv, X. Chen, D. Cao, and Y. Chen, "A personalized behavior learning system for human-like longitudinal speed control of autonomous vehicles," Sensors (Basel, Switzerland), vol. 19, no. 17, pp. 3671-3690, 2019.

[9] Y. Xing, C. Lv, and D. Cao, "Personalized vehicle trajectory prediction based on joint time series modeling for connected vehicles," IEEE Transactions on Vehicular Technology, vol. 69, no. 2, pp. 1341-1352, 2019.

[10] Y. Xing, C. Lv, H. Wang et al., "Driver lane change intention inference for intelligent vehicles: framework, survey, and challenges," IEEE Transactions on Vehicular Technology, vol. 68 , no. 5 , pp. 4377-4390, 2019.

[11] C. Lv, X. Hu, A. Sangiovanni-Vincentelli, Y. Li, C. M. Martinez, and D. Cao, "Driving-style-based codesign optimization of an automated electric vehicle: a cyber-physical system approach," IEEE Transactions on Industrial Electronics, vol. 66, pp. 2965-2975, 2018.

[12] N. Kuge, T. Yamamura, O. Shimoyama, and A. Liu, "A driver behavior recognition method based on a driver model framework," SAE Technical Paper, vol. 109, no. 6, pp. 469-476, 2000.

[13] M. Quintero, J. Lopez, and A. C. C. Pinilla, "Driver behavior classification model based on an intelligent driving diagnosis system," in Proceedings of the 2012 15th International IEEE Conference on Intelligent Transportation Systems, pp. 894-899, Anchorage, Alaska, USA, September 2012.
[14] G. Zhenhai, S. Tianjun, and H. Lei, "A multi-mode control strategy for EV based on typical situation," SAE Technical Paper, vol. 1, pp. 1-8, 2017.

[15] H.-S. Ahn, Y. Chen, and K. L. Moore, "Iterative learning control: brief survey and categorization," IEEE Transactions on Systems, Man and Cybernetics, Part C (Applications and Reviews), vol. 37, no. 6, pp. 1099-1121, 2007.

[16] R. Chi, D. Wang, Z. Hou, and S. Jin, "Data-driven optimal terminal iterative learning control," Journal of Process Control, vol. 22, no. 10, pp. 2026-2037, 2012.

[17] M. M. Minderhoud and P. H. L. Bovy, "Extended time-tocollision measures for road traffic safety assessment," Accident Analysis \& Prevention, vol. 33, no. 1, pp. 89-97, 2001.

[18] W. Wachenfeld, P. Junietz, R. Wenzel, and H. Winner, "The worst-time-to-collision metric for situation identification," in Proceedings of the Intelligent Vehicles Symposium (IVS), pp. 356-362, Gotenburg, Sweden, September 2016.

[19] L. Zhang, H. Ding, J. Shi et al., "An adaptive backstepping sliding mode controller to improve vehicle maneuverability and stability via torque vectoring control," IEEE Transactions on Vehicular Technology, vol. 69, no. 3, pp. 2598-2612, 2020. 